Supporting Information

\title{
How to Choose a Secondary Interaction to Improve Stretchability of Associative Polymers?
}

\author{
Huanhuan Yang ${ }^{1,2}$, Shilong $\mathrm{Wu}^{1}$, and Quan Chen ${ }^{1,2^{*}}$
}

${ }^{1}$ State Key Laboratory of Polymer Physics and Chemistry, Changchun Institute of Applied

Chemistry, Chinese Academy of Sciences, 130022 Changchun, China

${ }^{2}$ University of Science and Technology of China, 230026 Hefei, China

*Email: qchen@ciac.ac.cn

$\underline{\text { Table of contents: }}$

1. Synthesis

1.1 Synthesis of monomer 3-bromopropyl methacrylate

1.2 Synthesis of monomer 3-(thymin-1-yl) propyl methacrylate

1.3 Synthesis of monomer 2-(2-ureido-4[1H]-6-methylpyrimidinone) ethyl methacrylate (UPy-MA)

2. Infrared Spectroscopy

3. DSC Traces

4. Linear Viscoelasticity (LVE)

4.1 LVE of single-network samples and pure PHMA at $T_{\mathrm{r}}=T_{\text {iso }}$

4.2 LVE of single-network sample Na-4.1 and three double-network samples $2 \mathrm{H}$ -

$5.5 \& \mathrm{Na}-4.0,3 \mathrm{H}-6.2 \& \mathrm{Na}-4.6$ and $4 \mathrm{H}-6.0 \& \mathrm{Na}-4.5$

4.3 Shift factors of all single-network and double-network samples

5. Extensional Rheology 


\section{Synthesis}

1.1 Synthesis of monomer 3-bromopropyl methacrylate<smiles>C=C(C)C(=O)OCCCBr</smiles>

Scheme S1: The synthesis route of monomer 3-bromopropyl methacrylate

3-bromopropyl methacrylate was prepared based on the method reported in literature ${ }^{1}: 0.05$ mol 3-bromo-1-propanol $(6.95 \mathrm{~g})$ and $0.056 \mathrm{~mol}$ triethylamine $(5.67 \mathrm{~g})$ were dissolved in 100 $\mathrm{mL}$ DCM, and then methacryloyl chloride was added dropwise to the reaction mixture immersed in ice bath. After one day's reaction at room temperature, the reaction was quenched by the addition of $10 \mathrm{~mL}$ methanol and stirred for $30 \mathrm{~min}$. After that, $100 \mathrm{~mL}$ saturated aqueous $\mathrm{NaHCO}_{3}$ was added. The raw product was washed three times with deionized water and dried with anhydrous $\mathrm{MgSO}_{4}$ for 15 min until the product became pink-colored.

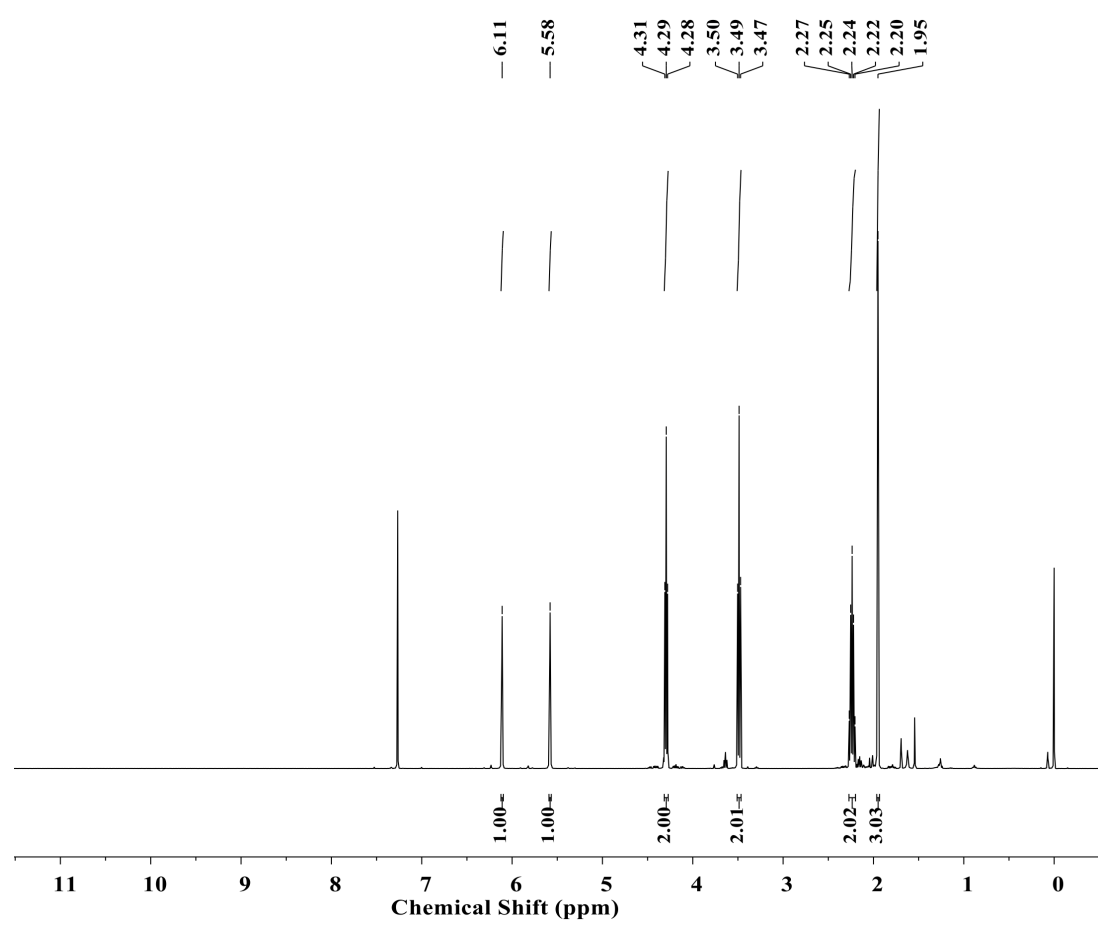

Figure S1: ${ }^{1} \mathrm{H}-\mathrm{NMR}$ spectrum of monomer 3-bromopropyl methacrylate. 
After the filtration and concentration, the desired product of 3-bromopropyl methacrylate can be obtained via further purification using column chromatography $(10 \%$ ethyl acetate in $n$ hexane), and the result of ${ }^{1} \mathrm{H}-\mathrm{NMR}$ spectrum is shown in Figure S1. ${ }^{1} \mathrm{H}-\mathrm{NMR}(400 \mathrm{MHz}$, $\left.\mathrm{CDCl}_{3}\right): \delta(\mathrm{ppm}) 6.11(\mathrm{~s}, 1 \mathrm{H}), 5.58(\mathrm{~s}, 1 \mathrm{H}), 4.28-4.31(\mathrm{t}, 2 \mathrm{H}), 3.47-3.5(\mathrm{t}, 2 \mathrm{H}), 2.20-2.27(\mathrm{~m}$, $1 \mathrm{H}), 1.95(\mathrm{~s}, 3 \mathrm{H})$.

1.2 Synthesis of monomer 3-(thymin-1-yl) propyl methacrylate<smiles>C=C(C)C(=O)OCCCBr</smiles><smiles>Cc1c[nH]c(=O)[nH]c1=O</smiles>

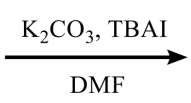<smiles>C=C(C)C(=O)OCCCn1cc(C)c(=O)[nH]c1=O</smiles>

Scheme S2: The synthesis route of monomer 3-(thymin-1-yl) propyl methacrylate

3-(thymin-1-yl) propyl methacrylate was prepared according to the literature ${ }^{1}: 28.6 \mathrm{mmol}$ dry $\mathrm{K}_{2} \mathrm{CO}_{3}(3.96 \mathrm{~g}), 1.80 \mathrm{mmol}$ tetrabutylammonium iodide (0.66g) and $19.5 \mathrm{mmol}$ 3-bromopropyl

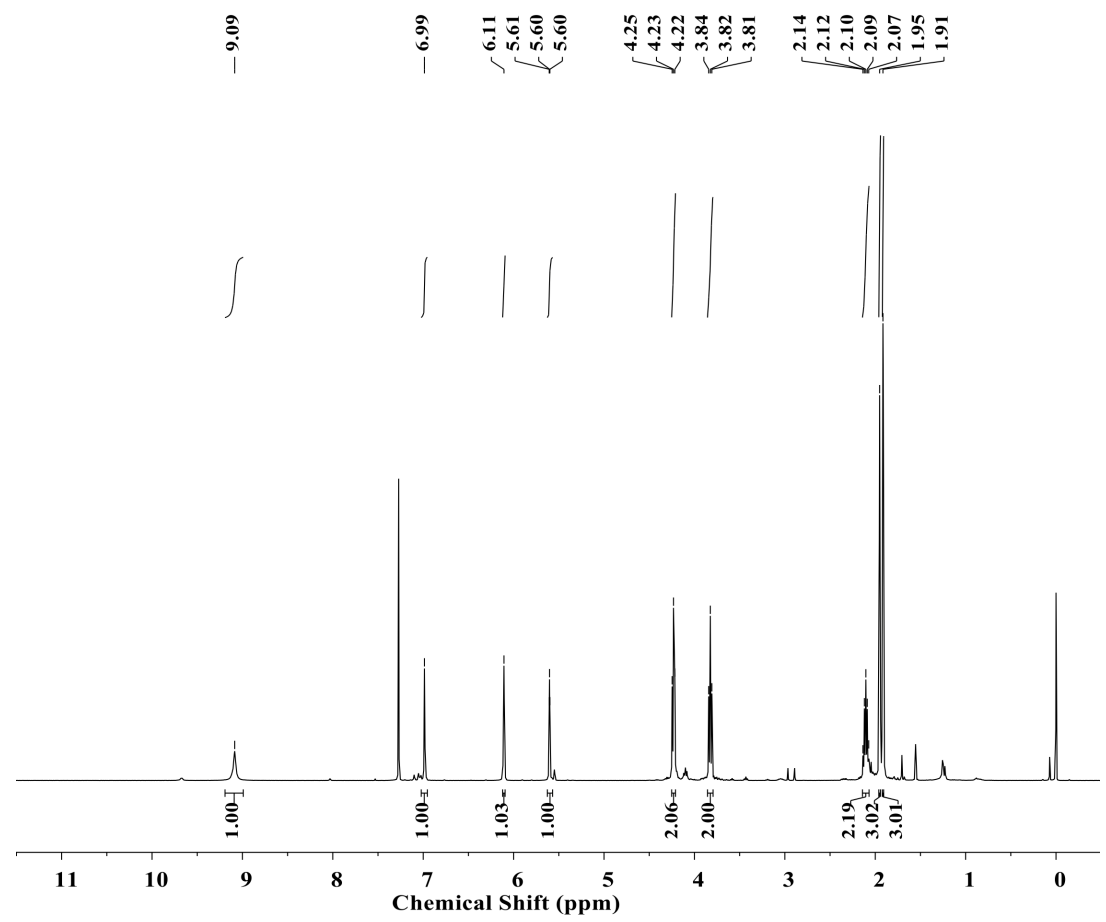

Figure S2: ${ }^{1} \mathrm{H}-\mathrm{NMR}$ spectrum of monomer 3-(thymin-1-yl) propyl methacrylate. 
methacrylate $(4.04 \mathrm{~g})$ were added to the solution of $28.4 \mathrm{mmol}$ thymine $(3.58 \mathrm{~g})$ in $200 \mathrm{~mL}$ DMF. The white suspension was reacted for about 2 days at room temperature, and then distillated under reduced pressure to remove DMF. The crude product was purified twice using the column chromatography (10\% methyl alcohol in DCM), the white-solid product was obtained after drying. The result of ${ }^{1} \mathrm{H}-\mathrm{NMR}$ spectrum is shown in Figure S2. ${ }^{1} \mathrm{H}-\mathrm{NMR}(400 \mathrm{MHz}$, $\left.\mathrm{CDCl}_{3}\right): \delta(\mathrm{ppm}) 9.09(\mathrm{~s}, 1 \mathrm{H}), 6.99(\mathrm{~s}, 1 \mathrm{H}), 6.11(\mathrm{~s}, 1 \mathrm{H}), 5.60-5.62(\mathrm{t}, 1 \mathrm{H}), 4.22-4.25(\mathrm{t}, 2 \mathrm{H})$, 3.81-3.84 (t, 2H), 2.07-2.14 (m, 2H), $1.95(\mathrm{~s}, 3 \mathrm{H}), 1.91(\mathrm{~s}, 3 \mathrm{H})$.

1.3 Synthesis of 2-(2-ureido-4[1H]-6-methylpyrimidinone) ethyl methacrylate (UPy-MA)<smiles>C=C(C)C(=O)COC(=O)Cn1c(C)cc(=O)nc1NCCOC(=O)NCCOC(=O)C(=C)C</smiles>

Scheme S3: The synthesis route of monomer 2-(2-ureido-4[1H]-6-methylpyrimidinone) ethyl methacrylate (UPy-MA)

UPy-MA was synthesized according to the method reported in literature ${ }^{2}: 32 \mathrm{mmol} 2$-amino4-hydroxy-6-methylpyrimidine $(4 \mathrm{~g})$ and $50 \mathrm{~mL}$ dry DMSO were added to a tube at the temperature of $170^{\circ} \mathrm{C}$. When the solid was completely dissolved, the oil bath was removed and $35.4 \mathrm{mmol} 2$-isocyanatoethyl methacrylate $(5.5 \mathrm{~g})$ was added dropwise into the tube under vigorous stirring. The reaction was quenched quickly using cold water to inhibit the polymerization. The white-solid precipitation was washed by acetone three times to remove the unreacted reactants and DMSO, and then vacuum-dried at $50^{\circ} \mathrm{C}$ for about 2 days prior to the measurements. The result of ${ }^{1} \mathrm{H}-\mathrm{NMR}$ spectrum is shown in Figure S3. ${ }^{1} \mathrm{H}-\mathrm{NMR}(400 \mathrm{MHz}$, 
$\left.\mathrm{CDCl}_{3}\right): \delta(\mathrm{ppm}) 12.97(\mathrm{~s}, 1 \mathrm{H}), 11.94(\mathrm{~s}, 1 \mathrm{H}), 10.43(\mathrm{~s}, 1 \mathrm{H}), 6.18(\mathrm{~s}, 1 \mathrm{H}), 5.79(\mathrm{~s}, 1 \mathrm{H}), 5.55(\mathrm{~s}$, 1H), 4.26-4.29 (t, 2H), 3.56-3.59 (m, 2H), $2.24(\mathrm{~s}, 3 \mathrm{H}), 1.93(\mathrm{~s}, 3 \mathrm{H})$.

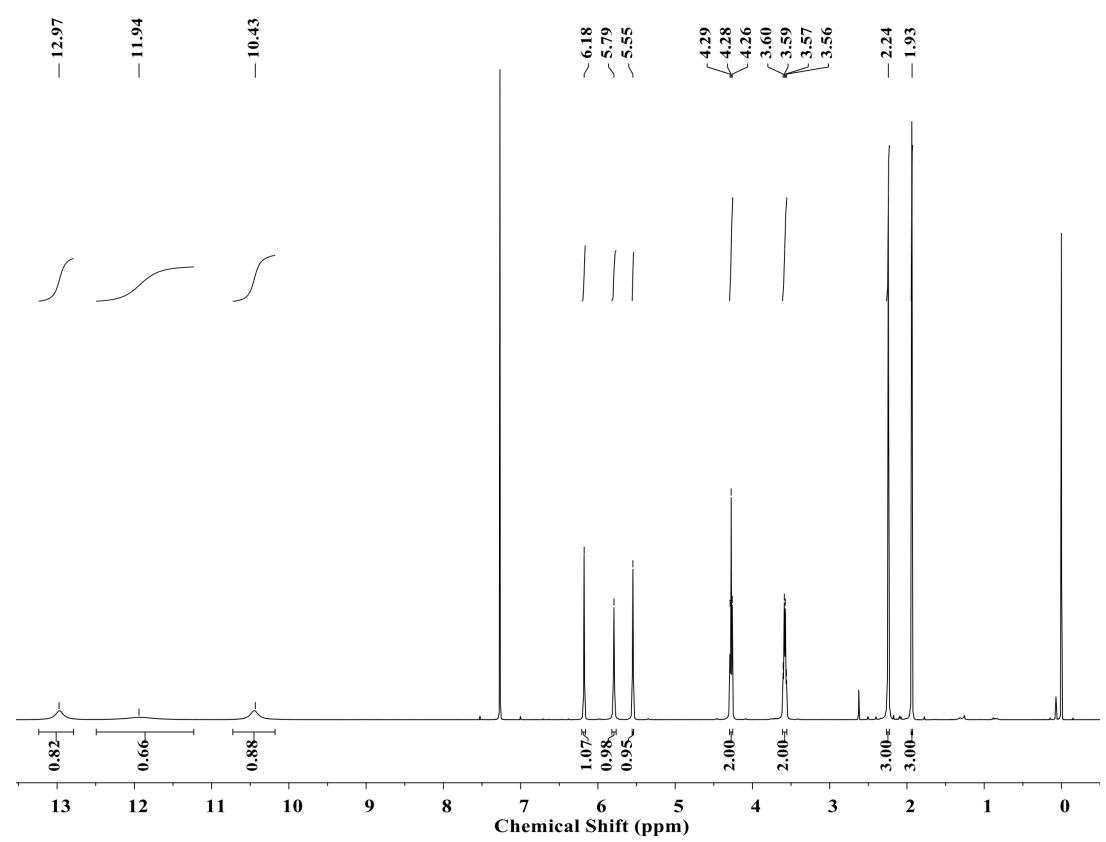

Figure S3: ${ }^{1} \mathrm{H}-\mathrm{NMR}$ spectrum of monomer 2-(2-ureido-4[1H]-6-methylpyrimidinone) ethyl methacrylate (UPy-MA). 


\section{Infrared spectroscopy}

Temperature-dependent Fourier transform infrared (FTIR) spectra were recorded in real time on a Brucker VERTEX-70 spectrometer equipped with a temperature-control chamber from room $T$ to the highest $T$ of the LVE measurements. The sample was loaded on a $\mathrm{KBr}$ window and placed in a chamber for the measurements.
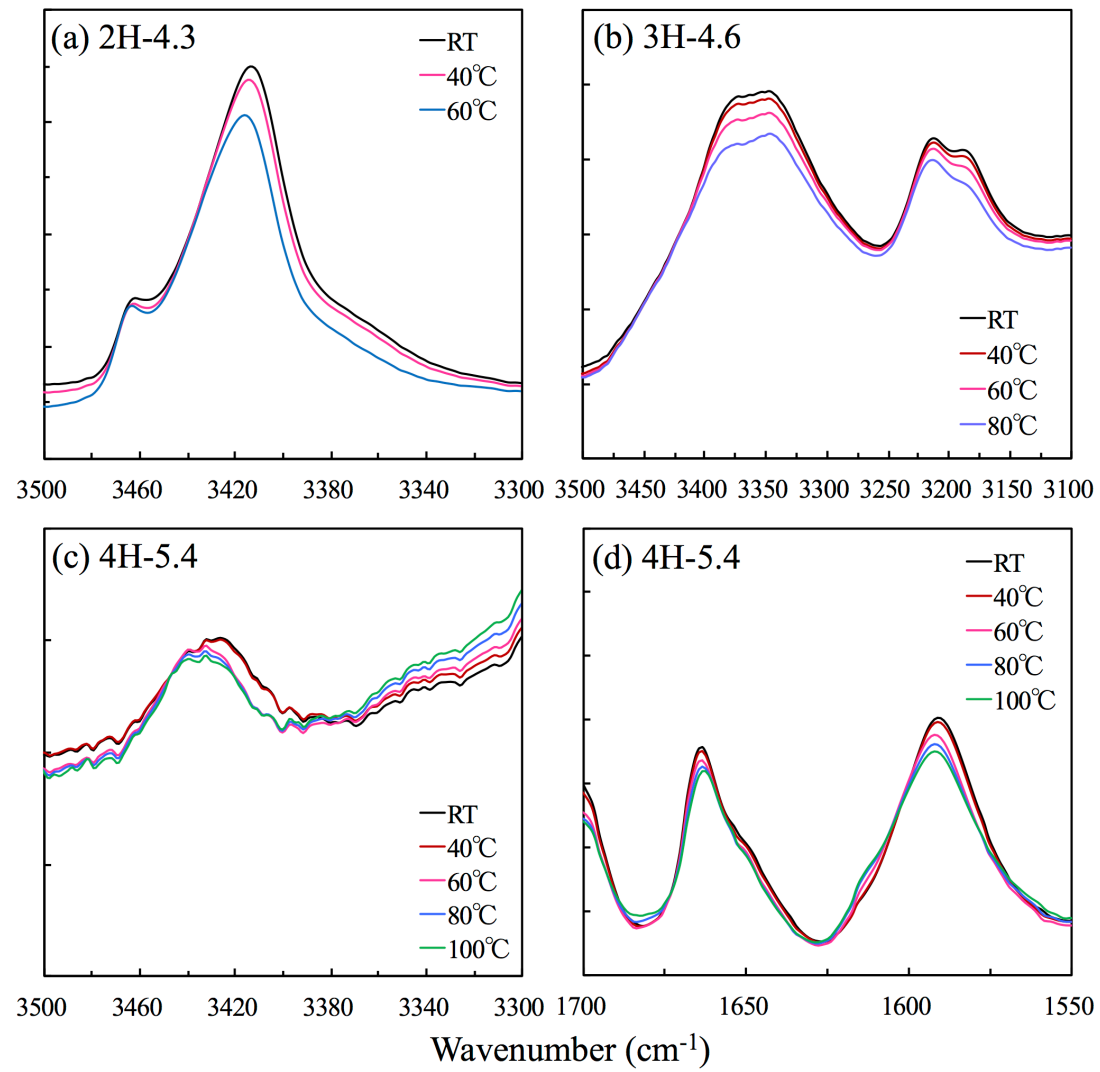

Figure S4: FTIR spectra at various temperature $(a, b, c)$ in the $\mathrm{N}-\mathrm{H}$ stretching region for (a) $2 \mathrm{H}-4.3$, (b) $3 \mathrm{H}-4.6$ and (c) $4 \mathrm{H}-5.4$, and (d) in the $1700-1500 \mathrm{~cm}^{-1}$ region showing the urea carbonyl and amide II bands of 4H-5.4.

Figures S4a-S4c compare the stretching peaks of N-H, "free" and hydrogen-bonded, obtained for $2 \mathrm{H}-4.3,3 \mathrm{H}-4.6$, and $4 \mathrm{H}-5.4$ at different $T$ as indicated. ${ }^{3}$ Figure S4d compares the absorption bands assigned to urea carbonyl $\left(\sim 1660 \mathrm{~cm}^{-1}\right)$ and the amide $I I\left(\sim 1580 \mathrm{~cm}^{-1}\right)$ group of UPy at 
different $T$ as indicated, ${ }^{4,5}$ where the absorption at $1580 \mathrm{~cm}^{-1}$ indicates the function groups of UPy are in dimerized form. ${ }^{6}$

From Figure S4a, we see that most of the N-H in $2 \mathrm{H}-4.3$ are hydrogen-bonded $\left(\sim 3415 \mathrm{~cm}^{-1}\right)$ and a small fraction is "free" $\left(\sim 3465 \mathrm{~cm}^{-1}\right)$ according to literature. ${ }^{7}$ With the temperature increasing, the relative intensity of the "free" N-H stretching peak increases slightly, whereas the $\mathrm{H}$-bonded one decreases and shifts to the higher wavenumber, indicative of weakening of the H-bonding at elevated temperature. However, this change is small and most of the N-H remain H-bonded. Indeed, our peaking fitting analysis using Lorentzian functions ${ }^{8}$ suggests that the fraction of "free" N-H bonds is less than $10 \%$ at room $T$ and increases by a mere $\sim 1 \%$ at $60^{\circ} \mathrm{C}$. This trend may explain the approximate validity of the tTs of LVE in the same $T$ range. For $3 \mathrm{H}-4.6$ and $4 \mathrm{H}-5.4$, the hydrogen bonds should be even stronger. In accordance with this expectation, the changes in the H-bonding state upon $T$ variation are also small for these two samples. 


\section{DSC Traces}

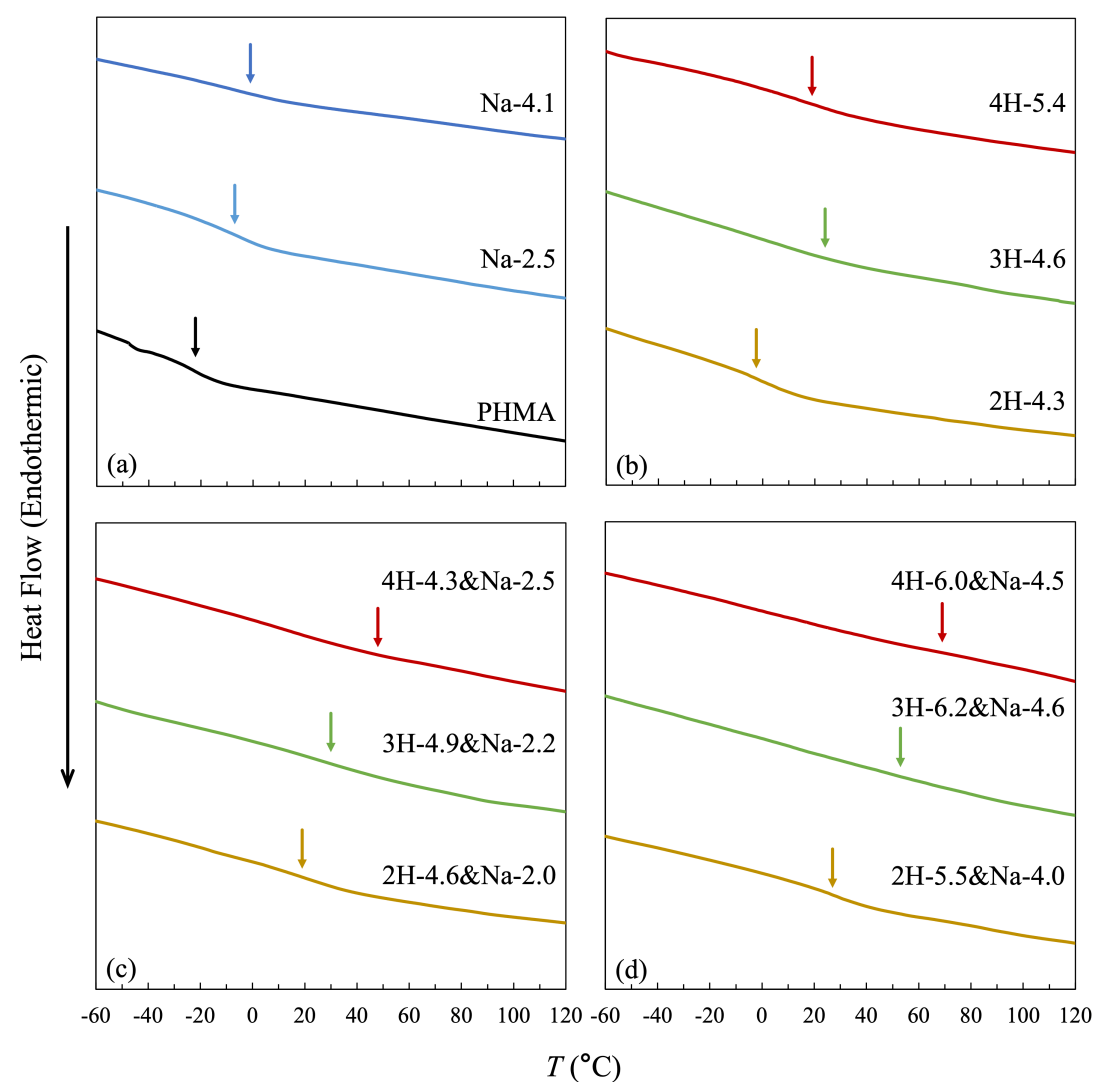

Figure S5: DSC traces of (a) pure PHMA and single-network samples containing only ionic groups, (b) single-network samples containing only hydrogen-bonding groups, and (c) doublenetwork samples for the lower ion contents and (d) double-network samples for the higher ion contents. 


\section{$4 \quad$ Linear Viscoelasticity (LVE)}

4.1 LVE of single-network samples and pure PHMA at $T_{\mathrm{r}}=T_{\text {iso }}$
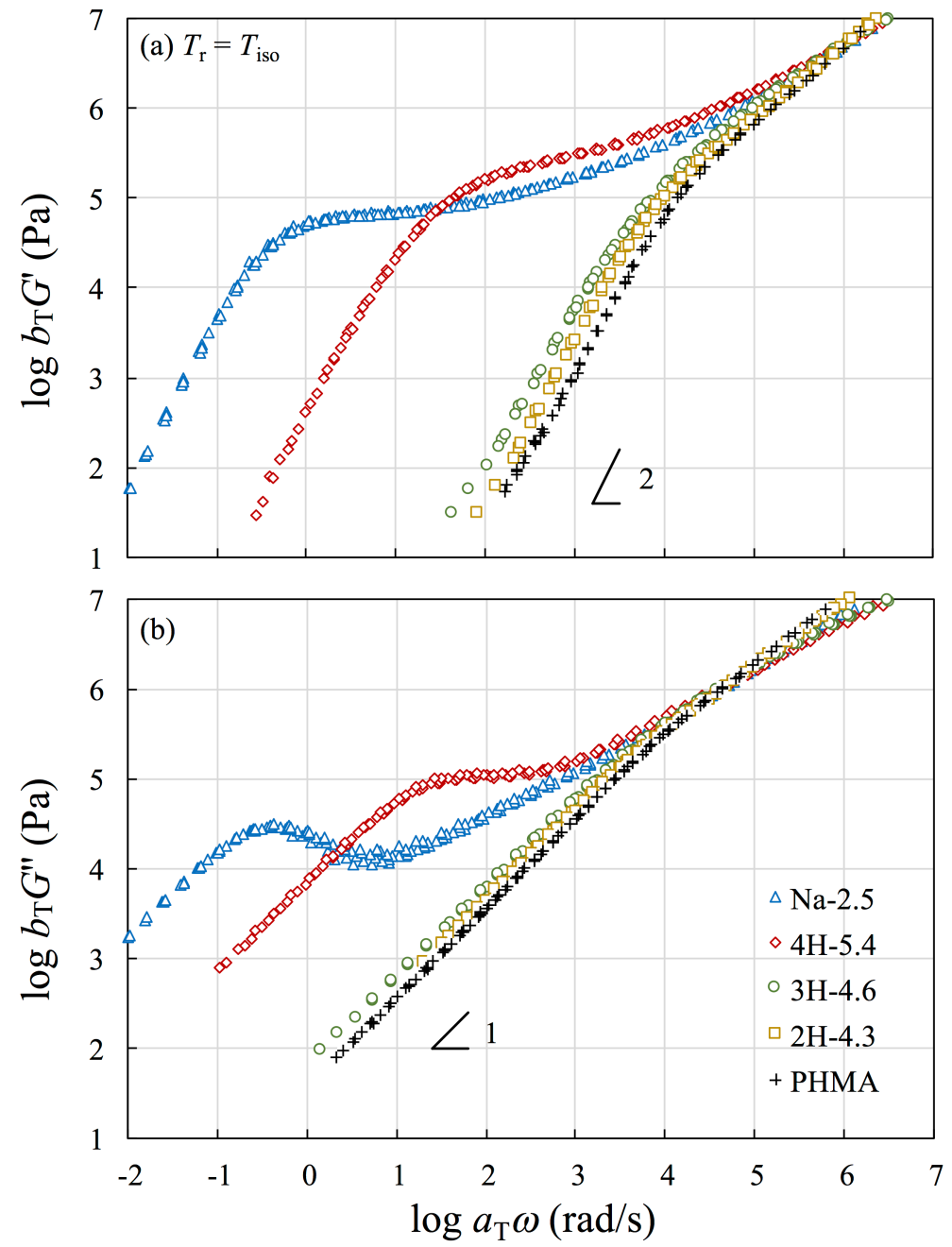

Figure S6: Comparison of the (pseudo-) master curves of the storage and loss moduli, $G^{\prime}$ and $G^{\prime \prime}$, obtained for pure PHMA, 2H-4.3, 3H-4.6, 4H-5.4, and Na-2.5. All (pseudo-) master curves are reduced at $T_{\mathrm{r}}=T_{\text {iso }}$. 
4.2 LVE of single-network sample Na-4.1 and three double-network samples $2 \mathrm{H}-5.5 \& \mathrm{Na}-$

4.0, 3H-6.2\&Na-4.6 and 4H-6.0\&Na-4.5.
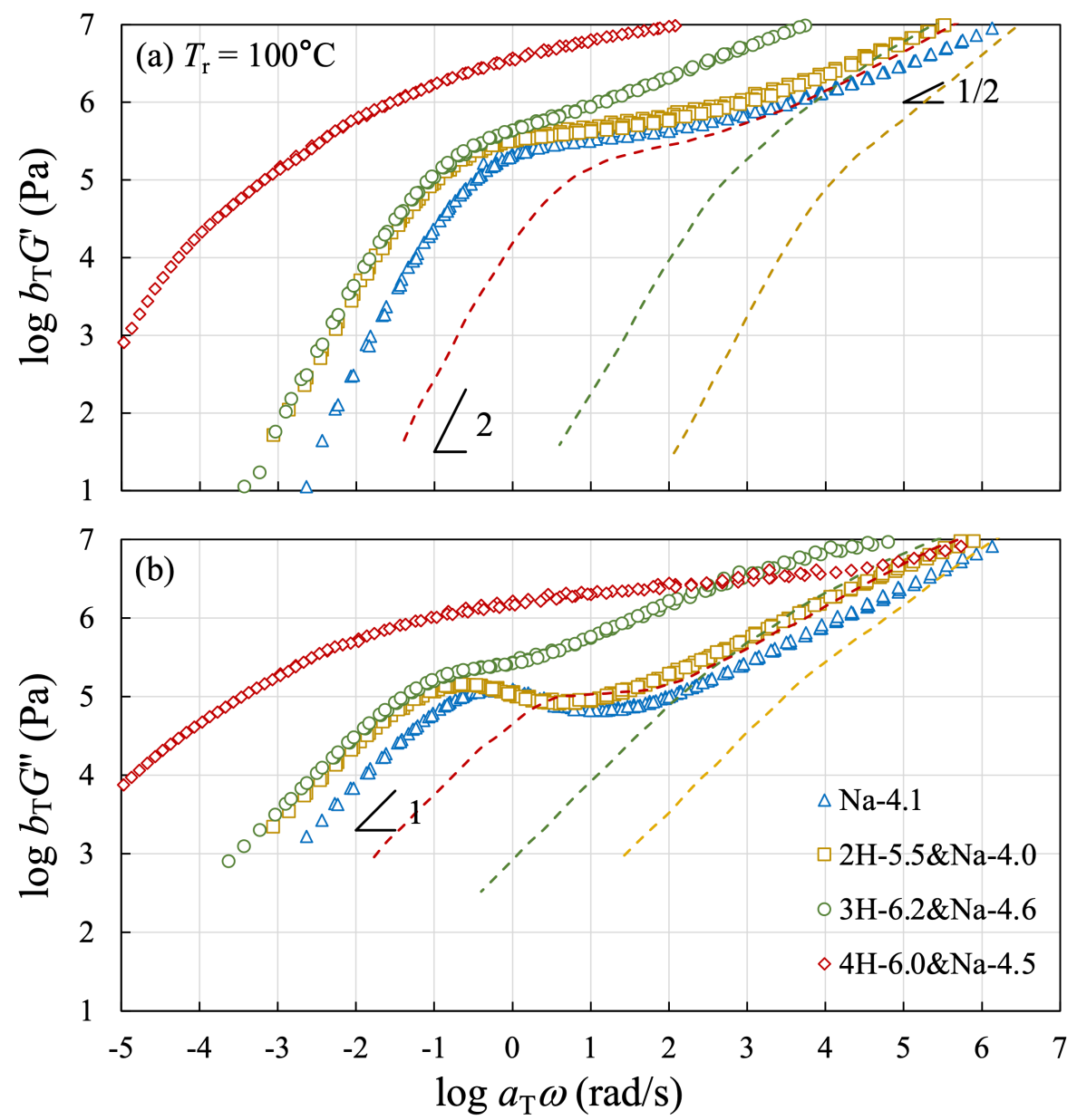

Figure S7: Comparison of the pseudo-master curves of the storage and loss moduli, $G^{\prime}$ and $G^{\prime \prime}$, obtained for single-network polymer Na-4.1(triangles), and three double-network samples $2 \mathrm{H}$ $5.5 \& \mathrm{Na}-4.0$ (squares), 3H-6.2\&Na-4.6 (spheres) and 4H-6.0\&Na-4.5 (diamonds). The dashed curves represent the modulus of the hydrogen-bonding samples shown earlier in Figure 2. All pseudo-master curves are reduced at $T_{\mathrm{r}}=100^{\circ} \mathrm{C}$. 
4.3 Shift factors of all single-network and double-network samples
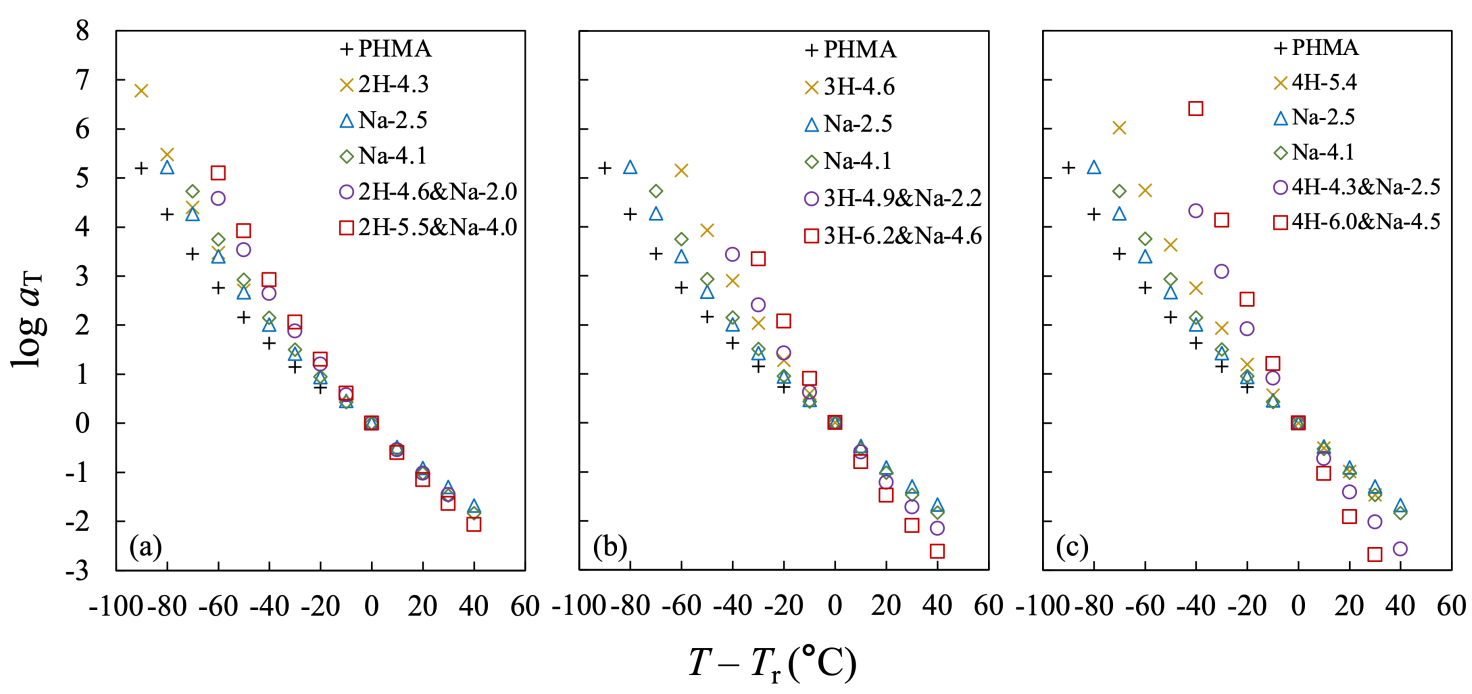

Figure S8: Comparison of logarithmic shift factors $\log a_{\mathrm{T}}$ plotted against $T-T_{\mathrm{r}}$ for (a) PHMA precursor and samples based on double hydrogen bonds or/and ionic groups, (b) PHMA precursor and samples based on triple hydrogen bonds or/and ionic groups, and (c) PHMA precursor and samples based on quadruple hydrogen bonds or/and ionic groups. 


\section{Extensional Rheology}

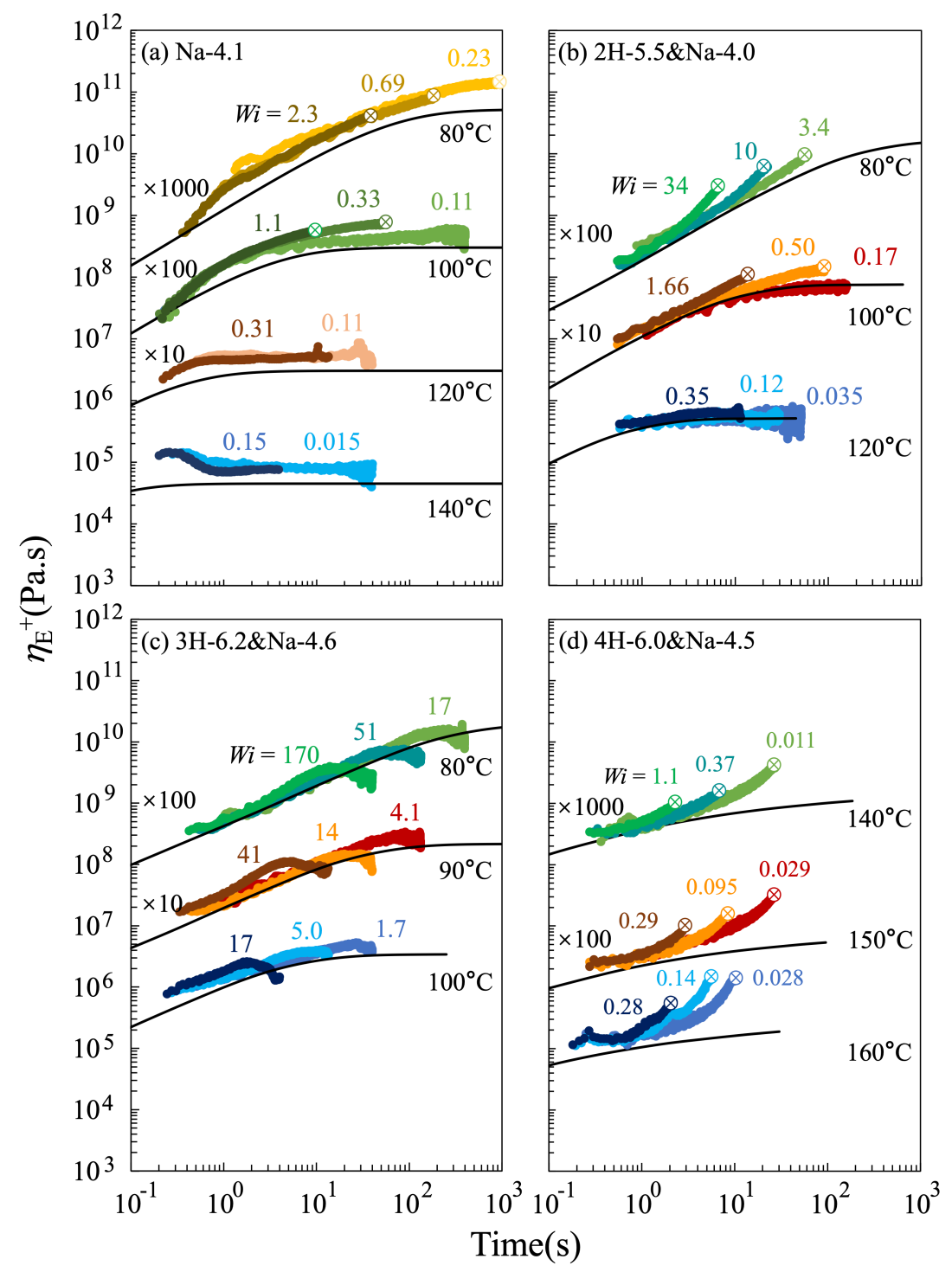

Figure S9: Plots of the extensional stress growth coefficient $\eta_{\mathrm{E}}{ }^{+}$against time at different temperatures $T$ for Na- $q$ and $\# \mathrm{H}-p \& N a-q$ samples shown in Figure S7. In each panel, the data at different temperatures are shifted vertically by factors as indicated (following the mark $\times$ ) to avoid overlap. The numbers therein show Weissenberg number $(\mathrm{Wi})$ defined according to the linear viscoelastic terminal relaxation time. The cross-marked symbol $(\otimes)$ indicates the sample fracture before reaching the preset strain $\left(\varepsilon_{\mathrm{H}}=4\right)$ during the measurement. 


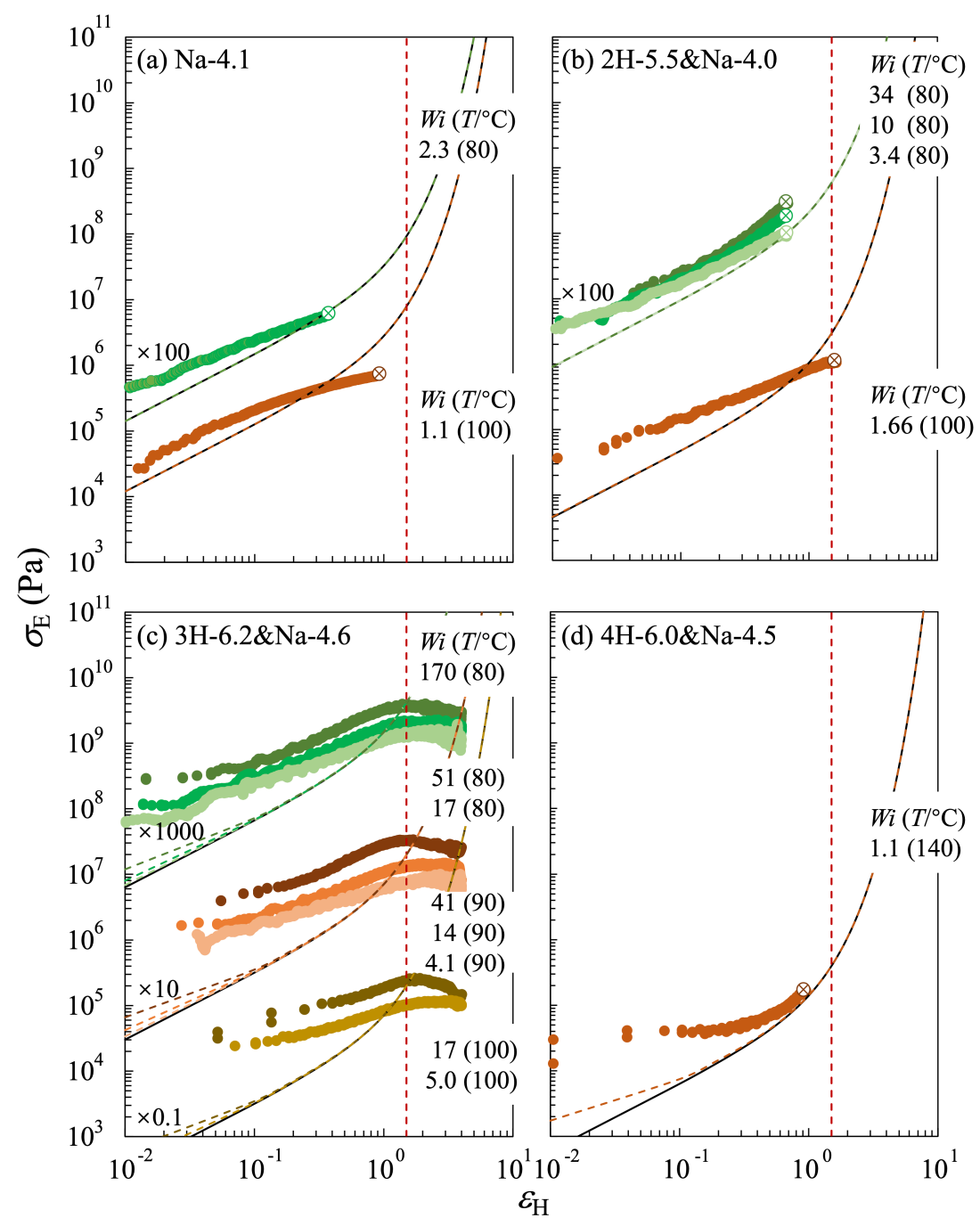

Figure S10: The true extensional stress $\sigma_{\mathrm{E}}$, plotted against Hencky strain $\varepsilon_{\mathrm{H}}$, for all measurements at $W i>1$ in Figure S9. The solid curves are expectation of the neo-Hookean model, and the red vertical dashed line shows $\varepsilon_{\mathrm{H}}=1.5$. The colored dashed curves are expected from a sum of the neo-Hookean model prediction and a dissipative part owing to the chain friction or dissociation/reassociation of hydrogen bonds. 


\section{References}

1. Spijker, H. J.; Dirks, A. J.; van Hest, J. C. M. Unusual Rate Enhancement in the Thymine Assisted ATRP Process of Adenine Monomers. Polymer 2005, 46 (19), 8528-8535.

2. Yamauchi, K.; Lizotte, J. R.; Long, T. E. Thermoreversible Poly(alkyl acrylates) Consisting of SelfComplementary Multiple Hydrogen Bonding. Macromolecules 2003, 36 (4), 1083-1088.

3. Skrovanek, D. J.; Howe, S. E.; Painter, P. C.; Coleman, M. M. Hydrogen-Bonding in Polymers - Infrared Temperature Studies of an Amorphous Polyamide. Macromolecules 1985, 18 (9), 1676-1683.

4. Lewis, C. L.; Anthamatten, M. Synthesis, Swelling Behavior, and Viscoelastic Properties of Functional Poly(Hydroxyethyl Methacrylate) with Ureidopyrimidinone Side-Groups. Soft Matter 2013, 9 (15), 4058-4066.

5. Appel, W. P. J.; Portale, G.; Wisse, E.; Dankers, P. Y. W.; Meijer, E. W. Aggregation of UreidoPyrimidinone Supramolecular Thermoplastic Elastomers into Nanofibers: A Kinetic Analysis. Macromolecules 2011, 44 (17), 6776-6784.

6. Creff, G.; Arrachart, G.; Hermet, P.; Wadepohl, H.; Almairac, R.; Maurin, D.; Sauvajol, J. L.; Carcel, C.; Moreau, J. J. E.; Dieudonne, P.; Man, M. W. C.; Bantignies, J. L. Investigation on the Vibrational and Structural Properties of a Self-Structured Bridged Silsesquioxane. Phys Chem Chem Phys 2012, 14 (16), 5672-5679.

7. Liu, Q. H.; Wang, C.; Guo, Y. H.; Peng, C.; Narayanan, A.; Kaur, S.; Xu, Y.; Weiss, R. A.; Joy, A. Opposing Effects of Side-Chain Flexibility and Hydrogen Bonding on the Thermal, Mechanical, and Rheological Properties of Supramolecularly Cross-Linked Polyesters. Macromolecules 2018, 51 (22), 9294-9305.

8. Matsumiya, Y.; Watanabe, H.; Urakawa, O.; Inoue, T.; Kwon, Y. Effect of Head-to-Head Association/Dissociation on Viscoelastic and Dielectric Relaxation of Entangled Linear Polyisoprene: An Experimental Test. Macromolecules 2020, 53 (3), 1070-1083. 